\title{
Le froid arrive, résistons, Collègues!
}

Marco Zellweger

Correspondance: Dr Marco Zellweger Psychiatre-Psychothérapeute FMH Avenue Reneveyres 6 Case postale 1133 CH-1110 Morges 1
Nombre d'entre nous se sont aperçus que ces dernières années, il ne fait pas bon être ni médecin, ni psychiatre. D'un côté, nous entendons chaque jour, dans le champ social, des discours qui revendiquent une amélioration des conditions de santé de la population et des procédures de soin: on parle de prévention, d'optimisation des prises en charge, de contrôle de qualité, et j'en passe. De l'autre côté, les médecins de terrain s'aperçoivent tous les jours qu'il devient toujours plus difficile de faire leur travail, en raison des contraintes administratives et financières auxquelles ils sont soumis.

Dans le domaine des troubles psychiques aussi, on reconnaît généralement l'importance des troubles mentaux et la nécessité d'y remédier: il est évident que ces troubles vont de pair avec un lourd tribut de souffrance pour le patient et son entourage, et de surcroît, ils occasionnent un poids financier très important.

Mais d'autre part, ces dernières années, les caisses-maladie et l'administration fédérale, ce que j'appellerai le «conglomérat administrativopolitico-financier», se sont fait les promoteurs d'un mouvement de méfiance envers les psychiatres: on ne veut plus les payer, on les harcèle en mettant en question l'utilisation de telle position du TARMED, l'économicité de leurs traitements et on leur demande le remboursement de sommes pharaoniques sous prétexte d'honoraires abusifs. Cela même, alors que les psychiatres et les pédopsychiatres touchent les honoraires les plus bas parmi tous les médecins, spécialistes ou médecins de premier recours.

Au cours de l'année 2006, nous avons tous pris connaissance que l'OFAS a voulu mettre en question le remboursement de la psychothérapie lors d'une procédure conduite à la hussarde, et a modifié le règlement qui détermine quelles sont les prestations qu'une caisse-maladie doit rembourser (les articles 2 et 3 de l'ordonnance sur les prestations de l'assurance des soins dite OPAS). La nouvelle version doit entrer en vigueur le $1^{\text {er }}$ janvier 2007. Je rappelle pour les lecteurs qui n'auraient pas suivi l'histoire, que cette nouvelle version exige que chaque traitement considéré comme une psychothérapie soit préalablement autorisé par la caisse-maladie, après préavis du médecin-conseil - mais la loi ne dit pas que la caisse est tenue de suivre sa proposition(!), ni ne prévoit de procédure de recours(!) - et que sa poursuite donne lieu à des autorisations qui doivent être régulièrement renouvelées.

Il est parfaitement clair que cette nouvelle législation crée les conditions cadre pour un rationnement de la psychothérapie, alors même que l'ancienne version de l'OPAS était restrictive et introduisait déjà une sorte de rationnement larvé.

Maintenant, perle sur le gâteau de Noël, nous apprenons que l'Office fédéral de la santé publique (OFSP) a approuvé à mi-décembre une procédure d'application de la norme en question, qu'il voudrait nous imposer. Il s'agirait d'utiliser un formulaire, élaboré par l'OFSP, dont le contenu me heurte beaucoup, car il va à l'encontre de certains principes mêmes qui régissent la conduite d'une psychothérapie.

En résumé, on voudrait que nous évaluions d'emblée la durée de la psychothérapie ellemême. Or, dans l'optique qui est la mienne, la conduite d'une psychothérapie va de pair avec le principe qu'il s'agit d'un processus qui va, et qui ne peut que se dérouler à sa vitesse propre, car chaque patient, chaque couple patient-psychothérapeute développe sa vitesse de travail et de changement singulière, que les deux acteurs ne connaissent pas d'emblée. Pour utiliser une métaphore, nous pourrions comparer cette situation à celle d'un couple de parents qui voudraient déterminer d'emblée, et à tout prix, le jour où leur enfant va commencer à marcher ou à prononcer ses premiers mots. Il n'est pas difficile d'imaginer l'effet qu'une telle attitude va produire. De même, nous pouvons déjà prévoir que ce procédé va avoir un effet d'enfermement et d'aliénation pour nos patients.

Plus encore, on voudrait associer le patient à cette démarche, en prévoyant qu'il contresigne le formulaire à son tour. On associe ainsi le patient à un savoir préalable à son sujet, puisqu'il est demandé au médecin qu'il formule le diagnostic le plus précis possible. A l'opposé, un des principes majeurs de la conduite d'une psychothérapie est de permettre au patient de construire son propre savoir à son sujet, et de pouvoir se déterminer plus librement sur ses désirs et ses choix.

De même, dans la conduite d'une psychothérapie, il s'agit pour le thérapeute d'être attentif à la place que les deux acteurs du processus interprètent tour à tour, le psychothérapeute étant 
toutefois soucieux de veiller à respecter strictement les limites de sa fonction, qui introduit et maintient une différenciation susceptible de permettre l'évolution du patient. Or, l'OFSP voudrait que notre rapport médical, qui finalement va apparaître comme l'acte inaugural de la psychothérapie, soit signé par les deux acteurs, qui se retrouvent pour ainsi dire au même niveau.

Dans le même sens, on voudrait encore que nous fixions un but préalable à la psychothérapie, alors que justement il s'agit, dans une psychothérapie, de suspendre tout but, pour instaurer un processus au cours duquel apparaît la capacité de mobiliser émotionnellement et symboliser les secteurs de sa personnalité en souffrance, qui s'expriment autrement par ses troubles, ses symptômes et éventuellement par sa maladie psychique.

Montrons donc que nous psychiatres-psychothérapeutes, que nous médecins savons aussi dire non et parfois nous montrer intraitables, comme les médecins de premier recours l'ont fait le $1^{\text {er }}$ avril 2006, et dirigeons le formulaire de l'OFSP à la seule place où il mérite de se retrouver, la poubelle. Continuons à rédiger nos rapports médicaux avec notre papier d'entête, en appliquant à la lettre le principe de la LaMal qui prévoit que l'on ne donne au médecin-conseil de la caisse que les informations strictement nécessaires à la vérification de l'adéquation du traitement appliqué. Nous ne sommes pas des employés de l'OFSP! Et n'ayons cure si l'on nous rétorque que l'on voudrait économiser des frais administratifs, ce n'est pas notre problème, ce n'est pas nous qui avons édicté ce règlement.

Notre politique professionnelle s'est déroulée jusqu'ici comme si le corps médical n'avait pas pris conscience du fait que nous sommes en état de guerre. Une guerre dont l'enjeu est le pouvoir de déterminer et contrôler les procédures de soins et les flux financiers qui s'y rapportent. Une guerre que nous pouvons comparer à une vraie maladie, même si elle n'existe pas dans la CIM10 ni dans ses versions futures, et dont l'origine se perd dans la nuit des temps, mais qui a pris une ampleur grandissante depuis une trentaine d'années, et qui peut très bien contaminer les médecins eux-mêmes, Brunner n'étant hélas pas le seul.

Une guerre qui se joue tout aussi bien sur un plan éthique, les technocrates voulant nous faire croire que la déontologie qui a inspiré les médecins dans la pratique de leur métier à travers les siècles, à savoir le respect et la promotion de la parfaite singularité de l'être humain, n'aurait aujourd'hui plus sa place. En lieu et place, nous assistons à la promotion d'une idéologie qui vou- drait gérer les processus de soin en se basant sur des procédés visant l'anonymat et l'uniformisation. Cette idéologie traduit la mise en œuvre d'un processus de désubjectivation. Elle utilise des modèles issus des sciences économiques, qui finissent par se retrouver, par contamination, dans nombre de concepts qui sont devenus hélas d'usage courant même pour nous médecins, comme le coût par cas, les guidelines, $l^{\prime}$ «evidence-based medicine», et j'en passe.

Notre politique professionnelle s'est jusqu'ici inspirée de la croyance qu'il suffirait de développer nos arguments nuancés pour que les technocrates nous écoutent. Il me semble que nous n'avons pas encore compris que nos alliés ne se trouvent pas parmi eux, mais au contraire parmi la population soucieuse de sa santé et du fait de bénéficier d'un système de soins performant. C'est donc à celle-ci qu'il nous faut nous adresser en toute priorité, et bien davantage que nous ne l'avons fait jusqu'ici. Autrement, quoiqu'en disent certains de nos représentants, nous irons d'échec en échec, obtenant au plus, comme cela a été souvent le cas jusqu'ici, d'être - parfois convoqués dans les salons bernois (je ne dis d'ailleurs pas qu'il ne faut pas y aller aussi), où l'on voudra aimablement nous attribuer $5 \mathrm{mi}$ nutes de parole dûment consignées au procèsverbal, alors que les dés sont pipés d'avance.

Créons donc du vent, Collègues, les politiques et les administratifs finissent toujours par se tourner dans sa direction!

Cessons cette politique qui n'amène qu'à des compromissions: nous nous sommes limités jusqu'ici à des protestations bien polies et timides dans nos cercles. Alors que tout autre organisation professionnelle non médicale, tout syndicat, après la modification des articles 2 et 3 de l'OPAS, aurait revendiqué l'abrogation de la nouvelle norme, la démission de $\mathrm{M}$. Couchepin et de son état-major, et œuvré immédiatement pour la mise en place d'un règlement adéquat, qui, en s'appuyant sur la reconnaissance de la psychothérapie en tant que méthode de traitement efficace, règle les modalités de sa prise en charge intégrale par les caisses-maladie.

Il s'agit davantage d'être conscient de notre force, qui découle de notre formation et de notre engagement pour nos patients, ainsi que de notre détermination à nous battre pour un exercice de la médecine qui garde sa vocation humaine. Boycottons donc le formulaire de l'OFSP, et chargeons nos représentants de faire connaître, de rendre visible, d'abord à la population elle-même, notre refus et d'en expliquer les raisons. 
De même, chargeons nos représentants d'étudier toutes les mesures de refus, sans à priori (j'en cite quelques unes: refus d'actes administratifs, refus de prise en charge, résiliation des conventions TARMED, utilisation accrue de procédures judiciaires, etc.) que nous pourrions faire valoir, voire utiliser dans notre combat. Elles témoignent de notre volonté et de notre engagement à défendre notre éthique, et signalent que nous ne sommes pas prêts à exercer notre profession n'importe comment. Et en cas de durcissement des conflits, étudions également toutes les mesures de solidarité que nous pourrions développer à l'égard des franges du corps médical impliquées.

Car notre salut, notre survie en tant que médecins libres praticiens, qui ne se sont pas laissés déposséder par les administrativo-politico- financiers, dépend de notre capacité de résistance et de notre engagement, quitte à devoir renoncer au confort douillet qui a été l'apanage des générations de nos prédécesseurs, et qui de toute manière se rétrécit comme peau de chagrin. En cas de conflit, il faut accepter de prendre des coups, voire de perdre. Ce n'est qu'à cette condition que l'on peut aussi gagner.

Collègues, le froid se lève, résistons. Inspirons nous de Momo [1] ou de Sam Lowry [2], et rappelons nous que s'il est minuit moins trois, il ne sera jamais minuit.

\section{Références}

1 Ende M. Momo. Stuttgart: Thienemanns; 1973.

2 Gilliam T. Brazil. Twentieth Century Fox; 1985. 